\title{
An Energy-Efficient Mobile-Controlled Vertical Handover Management for Real Time Services
}

\author{
Toan Nguyen-Duc, Eiji Kamioka \\ Graduate School of Engineering and Science, Shibaura Institute of Technology, Tokyo, Japan \\ Email: nb14501@shibaura-it.ac.jp,kamioka@shibaura-it.ac.jp
}

How to cite this paper: Nguyen-Duc, T. and Kamioka, E. (2016) An Energy-Efficient Mobile-Controlled Vertical Handover Management for Real Time Services. Journal of Computer and Communications, 4, 59-75. http://dx.doi.org/10.4236/jcc.2016.417004

Received: October 31, 2016

Accepted: December 26, 2016

Published: December 29, 2016

Copyright $\odot 2016$ by authors and Scientific Research Publishing Inc. This work is licensed under the Creative Commons Attribution International License (CC BY 4.0).

http://creativecommons.org/licenses/by/4.0/

\begin{abstract}
Multiple wireless access technology has been embedded into a single mobile device as a fundamental feature, aiming to give end users ubiquitous access at any time. To allow the users to enjoy the ubiquitous connectivity, the mobile device has to consume higher energy for the simultaneous activation of multiple wireless interface and the continuous connectivity. In addition, a seamless vertical handover between the access technologies is a mandatory requirement to insure the quality, reliability and continuity of real time services. In this paper, the continuity of real time services as well as energy saving for mobile devices has been taken into account. The conceptual idea is that whenever traffic rate is lower than a threshold it will be smoothly handed over to a low energy consumption technology, i.e., Bluetooth. When the traffic exceeds the limitation of Bluetooth bandwidth, it will be handed over to a wider bandwidth technology, i.e., Wi-Fi. In the considered scenarios, the technologies are not interconnected; hence, the vertical handover management must be fully controlled by the mobile devices. The performance of the system including energy saving and maintaining the continuity of real time services has been evaluated by direct measurements in a real testbed.
\end{abstract}

\section{Keywords}

Energy Efficient, Host-Controlled, Vertical Handover, Bluetooth, Wi-Fi, SDN Extension, Real Time Services

\section{Introduction}

The concurrent of multiple wireless access technology in a mobile device allows end users to be always best connected anywhere and anytime [1]. The users can use different access technologies to join their expected network and enjoy real 
time services. To enable this ubiquitous access, multiple wireless interface must be activated at all the time, waiting for their duties. Typically, when only one technology is used at any given time, the others can sleep. Although, the technologies, i.e., Wi-Fi, are in sleeping state, they still consume energy [2]. Besides, the continuous connectivity of real time services commonly requires the wireless interface to be awake to receive and send data. This will lead to higher energy consumption on the mobile device which is generally a battery-powered one. It is hence not a surprise that there is a huge research effort to reduce the energy consumed by the wireless communication. For instance, with reference to the Wi-Fi technology, several approaches tried to optimize the Power Save Mode (PSM) of the technology in order to increase the sleep period. These approaches did help to reduce energy consumption of a WLAN; however, they had to pay the cost of induced delay. Other attempts tried to eliminate the energy consumed during sleeping period by turning off the Wi-Fi interface [3] [4]. These works, however, require another technology, which is assumed to be interconnected with the WLAN network, to wake the Wi-Fi interface up. When the WiFi interface is off, there is no data in the network.

In this work, the unused wireless technologies are also turned off to save energy. In addition, when the bandwidth utilization is less than a threshold, all ongoing traffic are switched to a low energy consumption technology, i.e., Bluetooth. The process of changing the wireless technology, also called a vertical handover (VHO), must be seamless to support real time services. The VHO is considered as "seamless" if it is able to maintain all ongoing applications on the mobile device, offering both low VHO delay and small packet loss rate. Vertical handover techniques have been largely studied in a variety of domains including IP-based wireless networks [5]. Several techniques have been standardized such as Media Independent Handover (MIH) [6], Mobile IP [7] and Session Initiation Protocol (SIP) [8]. However, these approaches operate at the Network Layer or Transport Layer in the OSI model [9], hence, meet a long delay, i.e., $1500 \mathrm{~ms}$ [10], in the handover process due to the network overhead. To reduce the handover delay, Astorga [11] attempted to perform the handover at Data Link Layer. In Astorga's work, to support MIH for handover management, the device drivers of wireless interfaces have been modified. All the mentioned works above, however, require an additional network element, i.e., the decision engine in [11], to generate a handover trigger and gather the network topology information. When the networks are randomly formed, i.e., Wi-Fi adhoc network and Bluetooth scatternet, and are not interconnected to the others, the VHO must be performed totally by the mobile device.

To perform a seamless vertical handover between non-interconnected wireless networks, our former work [12] seems to be a potential candidate. In the proposed system, an energy-efficient algorithm was proposed to diminish the energy consumption on mobile devices. The devices cooperate with the others to at- 
tempt to switch all ongoing traffic, which are not sent to them as destinations, to Bluetooth to save energy. The feasibility of the proposed system has been confirmed by measurements on a real testbed. As an extension of our previous work, in this paper, a mobile-controlled VHO is proposed to support mobile devices to save energy and to maintain the continuity of real time services in non-interconnected wireless networks. The performance of the system is evaluated on a real testbed as well. The obtained results reveal that the real time service using UDP/ TCP traffic has been maintained. The minimal and maximal energy saving by the proposed system has also been estimated based on the measured values.

The rest of the paper is organized as follows: In Section 2, the theoretical background of vertical handover as well as the previous work is present. In Section 3, the detail of a mobile-controlled VHO management and the energy saving estimation will be explained. Section 4 will describe the evaluation on the performance of the system. Finally, the paper will be concluded in Section 5 .

\section{Vertical Handover in Heterogeneous Wireless Network}

\subsection{Theoretical Background}

A VHO occurs when the mobile device moves over heterogeneous access networks. The used access technology is changed along with the IP address in the $\mathrm{VHO}$, as the mobile nodes moves across different networks which use different access technology. [13]. The VHO procedure includes three phases: system discovery, VHO decision, and VHO execution [14]. As shown in Figure 1, in the first phase, all necessary information is gathered. The gathered information includes, but not limited to, the availability of nearby networks identified via $\mathrm{Re}$ ceived Signal Strength Indicator (RSSI), the throughput, the available bandwidth, the latency, the packet loss rate, the mobile device's state such as battery level, information of wireless interfaces. The collected information is, then, processed for making decisions in the next phase. In the second phase, the decision of when to trigger a handover and which network is selected will be made. The VHO needs to be triggered correctly to make an optimal handover, while the best network is selected to fulfil all requirements from applications or users. In the last phase, the handover is executed. This process is also called mobility management. In mobility management, there is commonly an entity that is in charge of controlling the execution process. Basically, the mobility management can be either network-controlled or mobile-controlled. In the network-controlled case, VHO is initiated and controlled by the network and the process can be assisted by the mobile device (mobile assisted). In the latter case, it is initiated and controlled by the mobile device. It can also be assisted by the network to make use of the information services.

\subsection{An Extended SDN Controller for Vertical Handover}

The control logic of an SDN-based wireless mobile device and the communica- 
tion from one controller to the other for managing the network, is given in Figure 2. On each device, a virtual interface, which is created by a software OpenFlow switch named OpenvSwitch (OVS) [15], is in charge of navigating traffic which goes through physical interfaces. The switch operates based on the rules defined by the original controller. The original controller here is a conventional SDN controller, which follows the specification given by the Open Networking Foundation [16]. Since the original controller is basically designed in a centralized manner in which it has a global view of the network topology, and thus it can provide rules for the switch easily. When the original controller is placed on a device, it becomes a local controller and faces a lack of network information. Therefore, an extended controller [17] has been introduced. The main task of the extended controller is to update network state in the local database to which the original controller can refer when necessary. Based on the stored information, the original controller is able to interpret the requirements from application

Handover execution phase

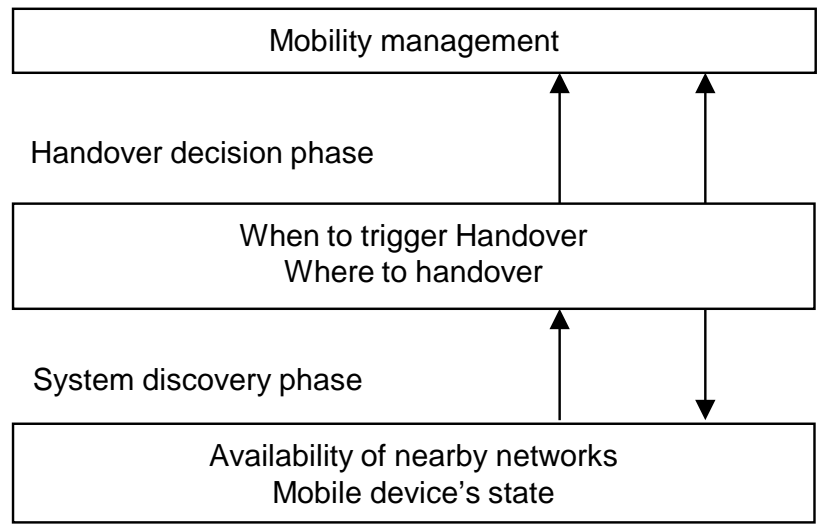

Figure 1. Handover management procedure.

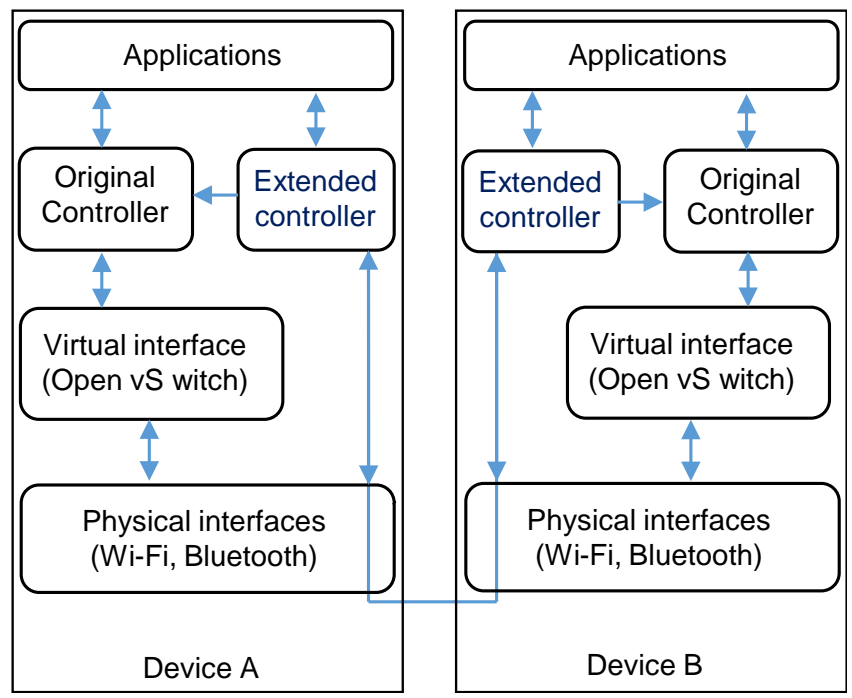

Figure 2. Controller to controller communication. 
into instructions for the virtual interface to navigate traffic correctly. When two devices, which are communicating with each other, need to change the access technology at the same time, i.e., for saving energy, they need to perform a VHO without external support. To this end, a controller to controller communication channel [12] has been developed. The extended controller on device A or B or both can listen to the running applications and tell the extended controller on the other's device. By doing that, two devices can perform VHO almost at the same time, reducing the handover delay.

\subsection{Previous Energy-Efficient Vertical Handover Algorithm}

In [12], an algorithm for triggering the energy-efficient vertical handover has been introduced. The detail of the algorithm is given in Figure 3. Firstly, the intermediate devices in the wireless multi-hop network receive the bandwidth requirement from the communicating devices. Based on the received information, the extended controller calculates the total required bandwidth Btotal. If Btotal is smaller than $1 \mathrm{Mbps}$, the extended controller will attempt to join a Bluetooth network. The Bluetooth network can be formed on the fly by the discussion of the extended controllers on the intermediate devices. If the device has already been using Bluetooth, it will maintain the connection. On the other hand, if Btotal is bigger than $1 \mathrm{Mbps}$, the extended controller will switch the connection to $\mathrm{Wi}-\mathrm{Fi}$. Also, if the $\mathrm{Wi}-\mathrm{Fi}$ is using, the $\mathrm{Wi}-\mathrm{Fi}$ connection will be retained.

\subsection{Drawback and Solution}

In the previous work [12], each SDN-based mobile device can perform a VHO from one technology (source technology) to the other (destination technology) without external help and can reduce energy consumption. However, when source and destination technology connect to non-interconnected networks, if

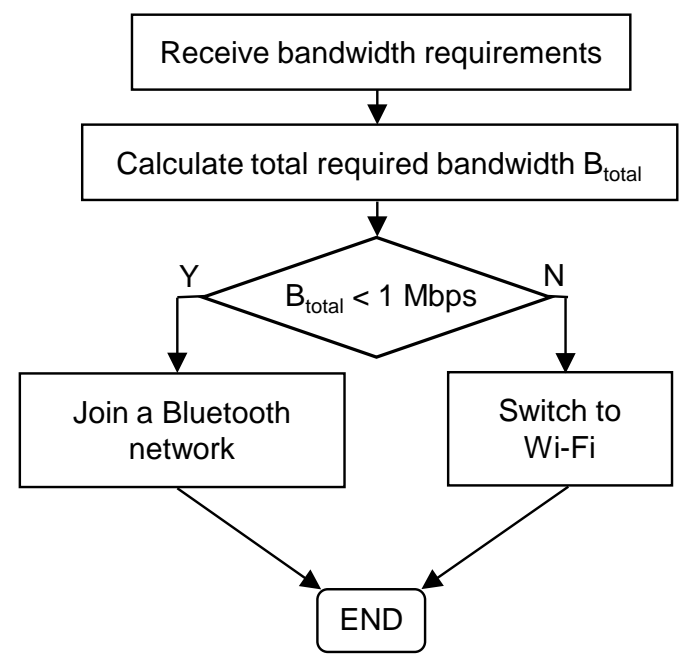

Figure 3. Triggering algorithm for the energy efficient vertical handover. 
two communicating devices complete the VHO process at different point of time, their connection will be interrupted. If the connection is interrupted in a long time, a lot of packets will be lost, hence, the quality of ongoing services cannot be guaranteed. To this end, in this work, a mobile-controlled VHO management is proposed to synchronize the VHO process on each device, minimizing the number packet loss to support real time services. The detail of the messages exchanged during the $\mathrm{VHO}$ process is also given and the performance of the system is validated by a set of experiment on a real testbed. Additionally, energy efficiency of the VHO management in various scenarios is confirmed by a direct measurement.

\section{An Energy-Efficient Mobile-Controlled Vertical Handover Management for Real Time Services}

\subsection{Assumed Network Environment}

The considered scenario in this work is shown in Figure 4. In the figure, two mobile devices, which are equipped with an extended controller described in the subsection 2.2, are communicating with each other. On each device, Wi-Fi and Bluetooth are available and the interference between them is assumed to be minimized. Basically, the devices use Wi-Fi technology to connect to the other via an access point (AP) or in an ad-hoc manner. Bluetooth technology is used as a backup one. It is also assumed that they are always able to join a Bluetooth network. However, the Wi-Fi network and Bluetooth network are not interconnected.

\subsection{Mobile-Controlled VHO Management}

The mobile-controlled VHO management process in this work, which follows a three-phase procedure discussed in the subsection 2.1, is illustrated in Figure 5. In the system discovery phase, the extended SDN controller, described in the subsection 2.2, on the mobile devices collects all the necessary information to make a decision whether or not to trigger VHO from the current access technology to the other. The collected information includes the local information of the device such as battery level, MAC and IP addresses as well as the information from the network side like access point properties, throughput,

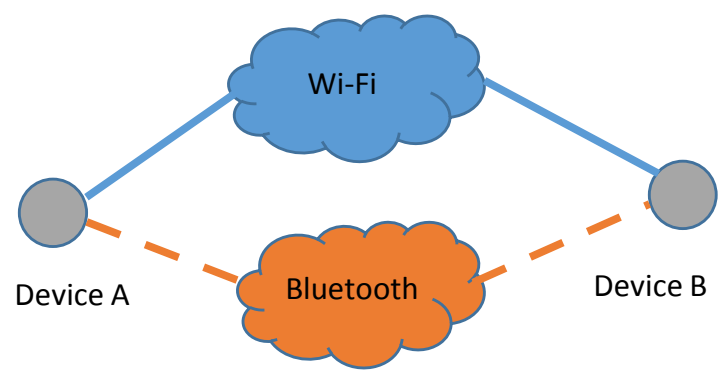

Figure 4. Considered network topology. 


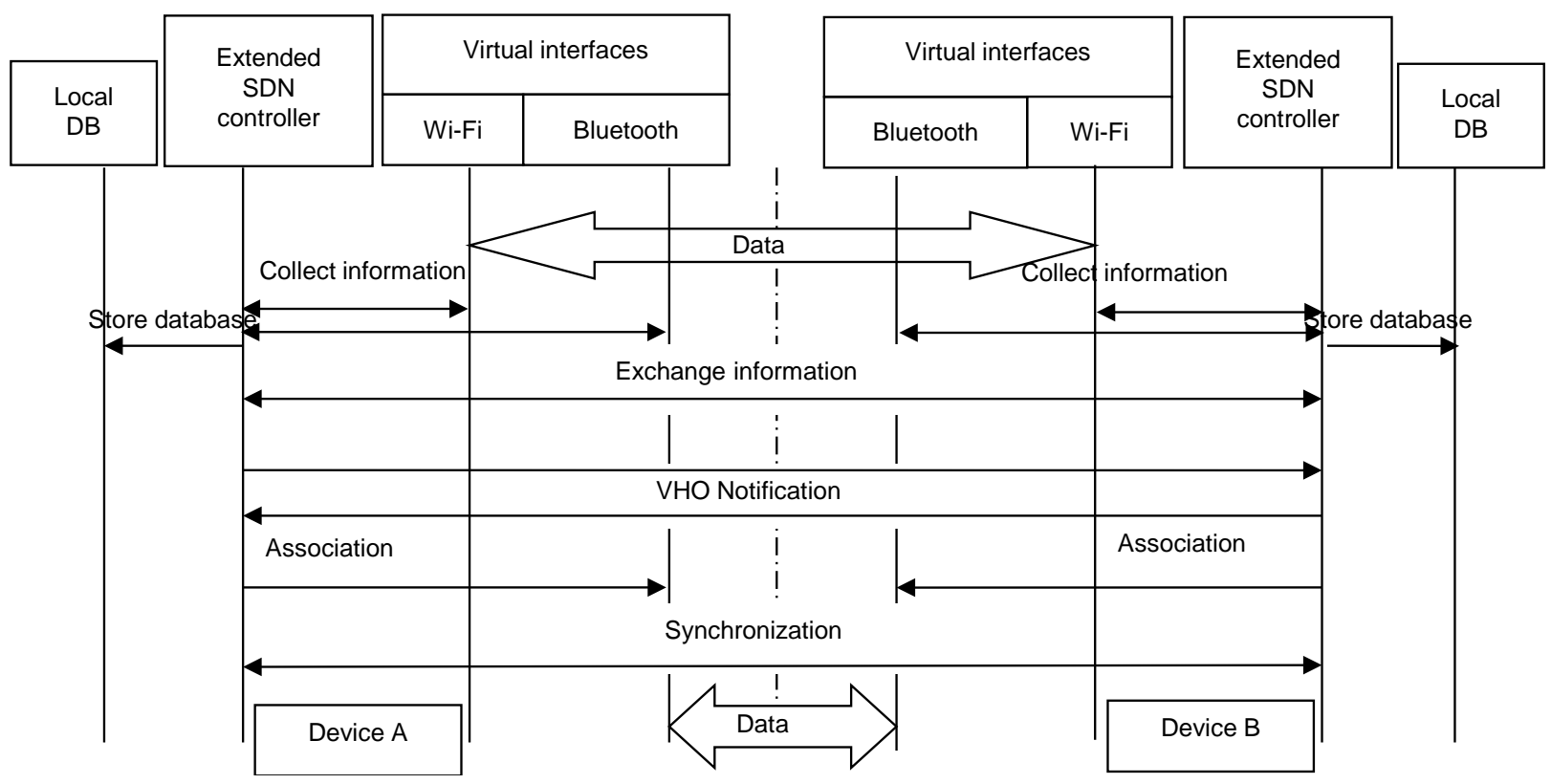

Figure 5. Messages exchanged in a mobile-controlled VHO management.

round-trip time (RTT). The information collected by each device is stored in a local database and is exchanged with other devices. If there is no updated information, they exchange the information only one time right after their communication starts. Both original SDN controller and extended SDN controller can retrieve information from the database. However, only the extended controller can add or modify the data in the database. Based on the collected information, the extended controller makes a decision whether or not to trigger a VHO in the second phase. In this work, the VHO decision is made simply based on the total throughput Btotal. If the Btotal is smaller than $1 \mathrm{Mbps}$, the extended controller will decide to perform a VHO from Wi-Fi to Bluetooth. Otherwise, it connects two devices via a Wi-Fi connection. When the VHO decision is made, the extended controller notifies the extended controller on the other device of the decision and which technology is going to be used. Once both sides receive the VHO notification, the VHO procedure starts. The extended controller activates and associates the backup wireless interface with its network. When the association process has finished, two devices must synchronize with the other in a similar way to the TCP 3-way handshake (SYN, SYN-ACK, and ACK). The synchronization process is to let the new wireless technology on two devices be available at almost the same time, hence, the packet loss will be reduced. After that, the extended controller can tell the original one to instruct the switch to navigate the traffic to the new interface.

\subsection{Energy Efficiency of the Mobile-Controlled VHO Management}

\subsubsection{Packet Transmission Time}

Since Wi-Fi and Bluetooth have different energy saving mechanism, it is neces- 
sary to identify how long the packets are sent over each technology to get the total energy consumption of the wireless communication. Let $N_{B}$ and $N_{W}$ denote the number of packets travelled over Bluetooth and Wi-Fi, respectively. Also let $R_{B}$ and $R_{W}$ denote the average data rate in the network of Bluetooth and $\mathrm{Wi}-\mathrm{Fi}$, respectively. The duration times used for Bluetooth and Wi-Fi are $T_{B}$ and $T_{W}$, respectively, and the system takes $T$ seconds to send the total $N$ packets as illustrated in Figure 6. The duration time $T_{B}$ and $T_{W}$ can be calculated by the following equations if there is no packet loss:

$$
\left\{\begin{array}{l}
N_{B}+N_{W}=N \\
N_{B} / R_{B}+N_{W} / R_{W}=T
\end{array}\right.
$$

From Equation (1) and (2), we can calculate TW and TB as follows:

$$
\left\{\begin{array}{l}
T_{W}=N_{W} / R_{W}=\left(R_{B} R_{W} T-R_{W} N\right) /\left(R_{B}-R_{W}\right) R_{W} \\
T_{B}=N_{B} / R_{B}=\left(R_{B} R_{W} T-R_{B} N\right) /\left(R_{W}-R_{B}\right) R_{B}
\end{array}\right.
$$

Let $P_{W}$ and $P_{B}$ denote energy consumption of Wi-Fi and Bluetooth. The total energy consumption of the wireless communication $P$ is expressed by Equation (5):

$$
P=P_{W} T_{W}+P_{B} T_{B}
$$

\subsubsection{The Number of Successful VHO}

In this work, the devices will try to switch their traffic from Wi-Fi to Bluetooth for saving energy while a service $S$ is running. The shorter Wi-Fi is used, the more energy is saved. It is assumed that the service $S$ lasts in $T$ seconds and Wi-Fi is initially used. The energy for running service $S$ is minimal if the device uses Wi-Fi in the shortest duration. The shortest time $T_{W \min }$ is achieved when the VHO process from Wi-Fi to Bluetooth and the service start at the same time. After VHO finishes, Bluetooth is used until service $\mathrm{S}$ ends. The $T_{W \min }$ is also the total execution time needed to perform a VHO from Wi-Fi to Bluetooth called $T_{V H O-W B}$. The minimal energy consumption $P_{\min }$ when using the mobile-controlled VHO management is calculated as follow:

$$
P_{\min }=P_{W} T_{W \min }+P_{B} T_{B}=P_{W} T_{V H O-W B}+P_{B} T_{B}
$$

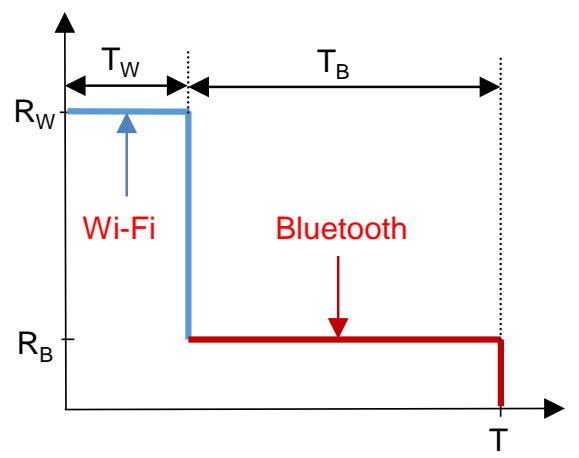

Figure 6. Packet transmission time on different technologies. 
Although the device can successfully perform the VHO from Wi-Fi to Bluetooth to save energy, it has to switch back to Wi-Fi in some cases. For example, if the required data rate of the communication is higher than Bluetooth capability, i.e., $1 \mathrm{Mbps}$, the device needs to switch the connection back to Wi-Fi to ensure the quality of running services. In the worst case, the device has to perform another $\mathrm{VHO}$ from Bluetooth to Wi-Fi right after finishing the handover from Wi-Fi to Bluetooth. In this case, the time period the device has been using Bluetooth corresponds to the total execution time to perform the VHO from Bluetooth to Wi-Fi (TVHO-BW). The maximal energy consumption Pmax when using the mobile-controlled VHO management is calculated as follow:

$$
P_{\max }=P_{W} T_{W}+P_{B} T_{B \min }=P_{W} T_{W}+P_{B} T_{V H O-B W}
$$

Since it takes $P_{W} T_{W}$ to perform a handover from Wi-Fi to Bluetooth and TVHO-BW to perform a handover in the inverted direction, the number of successful VHO when service $S$ still runs is the quotient in the division given in the Equation (8):

$$
N_{V H O}=T /\left(T_{V H O-W B}+T_{V H O-B W}\right)
$$

The minimal energy consumption when there is NVHO VHO occurred is:

$$
P_{N}=P_{W} T_{W}+P_{B} N_{V H O} T_{V H O-B W}
$$

\section{Performance Evaluation}

\subsection{Mobile-Controlled VHO Management Performance Evaluation}

This evaluation is to show the effectiveness of the mobile-controlled management when two SDN-based devices, at the same time, switch network traffic from Bluetooth to Wi-Fi and vice versa. The performance metrics are the packet loss rate and the handover delay.

\subsubsection{UDP Traffic Load}

To observe the handover delay and the packet loss rate, UDP is selected as the transport protocol because it provides a unidirectional communication, thus, lost packets are not retransmitted.

The testbed in this evaluation is illustrated in Figure 7. The figure shows that two SDN-based devices are placed within the communication coverage of a $\mathrm{Wi}-\mathrm{Fi}$ and a Bluetooth networks. In the evaluation, Iperf [17] is used to generate an UDP flow toward the direction from device A to device B.

In this work, the OVS is used to provide a virtual interface between an application and physical interfaces. Since the application only sees the virtual interface, it does not notice the change of physical interfaces. On the other hand, physical interfaces play the role of switch port. The OVS navigates traffic that comes in and goes out of the interfaces by OpenFlow rules, which are created by the SDN controller and are stored in OVS's flow table. The time taken to install OpenFlow rules is named $T_{\text {OFrules }}$. Note that the physical interface can be used 


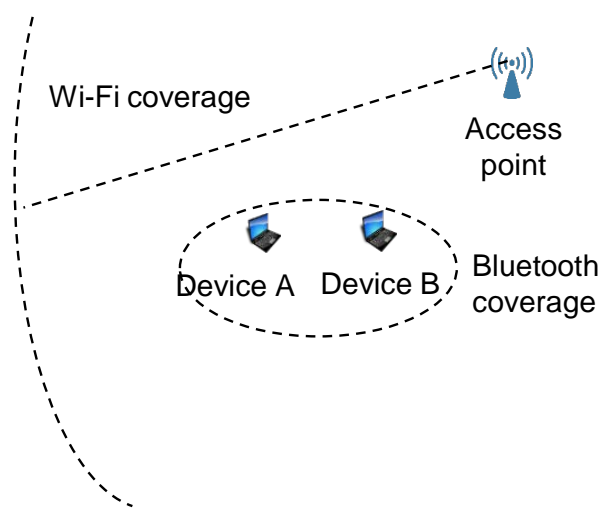

Figure 7. Experiment topology.

after it is activated and associated with its network. This processes takes $T_{N \text { Wonfig }}$ seconds. The different between the end time of configuring network and that of installing OpenFlow rules is defined as handover delay. The delay is the source of packet loss. For instance, if OVS finishes installing OpenFlow rules before the physical interface is configured, the traffic following the OpenFlow rules is sent to a physical interface, which has not been ready yet. Alternatively, if the new interface is ready, however, the OpenFlow rules is not installed, the traffic is not sent to it.

The measurement procedure is as follows: Firstly, device A with the role of a server starts Iperf in server mode and waits for incoming requests. On device B, an Iperf client then attempts to establish a connection with the Iperf server. While a $100 \mathrm{Kbps}$ UDP traffic is being sent, a VHO occurs randomly. The delays were captured at the same time with measuring the packet loss rate in the previous experiments. The procedure was repeated 50 times and the measured results are shown in Figure 8 and Figure 9. The Figure 8 shows that the network configuration duration $T_{B N W}$ and the OpenFlow installation duration $T_{B O F}$ of Bluetooth is $119.45 \mathrm{~ms}$ and $21.34 \mathrm{~ms}$ on average, respectively. The handover delay is as long as $98.11 \mathrm{~ms}$, causing 3.5\% packets losson average as shown in Figure 9. The packet loss rate is relatively high, however, mainly below $7 \%$, even if the traffic was switched from high data rate network to a lower one. In the in verted direction, a delay was inserted before executing install OpenFlow rules, reducing the handover delay to $18 \mathrm{~ms}$ on average. As a result, the packet loss is only $1 \%$ as given in Figure 9 . The obtained results confirm that the shorter delay is, the less packets are lost. Moreover, the handover delay can be reduced by reducing the different between $T_{W O F}$ and $T_{W N W}$. The handover delay is as small as $18 \mathrm{~ms}$ with $1 \%$ packet loss rate can support real time services.

\subsubsection{TCP Traffic Load}

The goal of this experiment is verified the feasibility of the proposed system in maintaining the continuity of the service. In addition, the obtained results will be used to calculate the total execution time to perform VHO from Wi-Fi to 


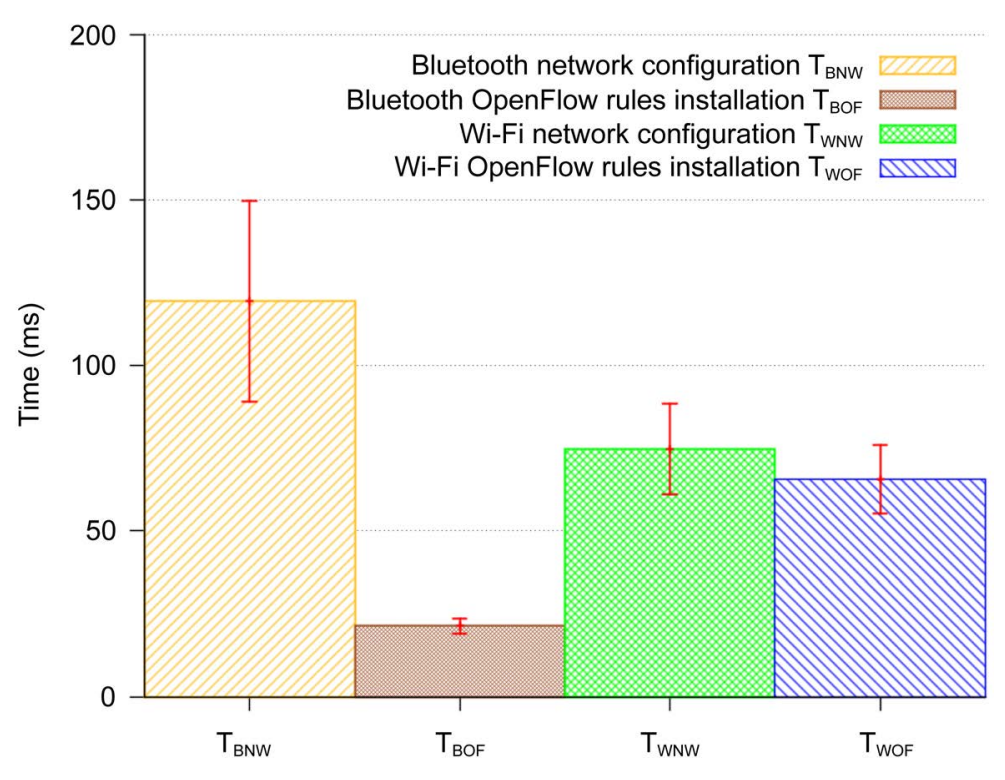

Figure 8. Handover delay measurement.

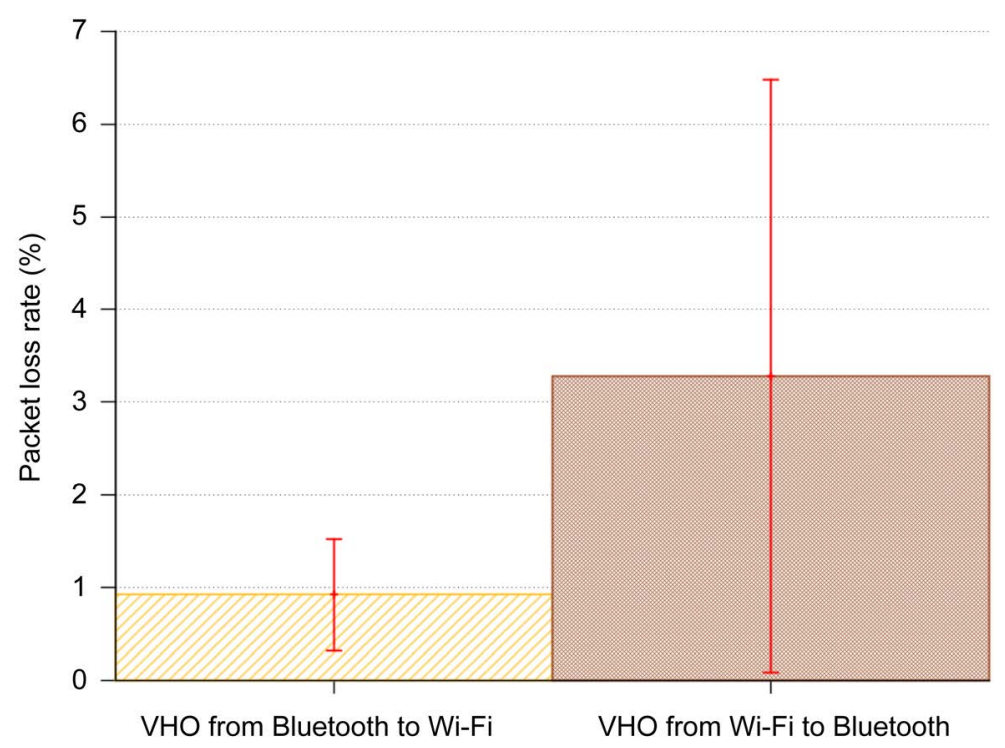

Figure 9. Packet loss rate when handover between Bluetooth and Wi-Fi.

Bluetooth and vice versa. To this end, a vertical handover during an FTP file exchange session has been observed. The average date rate of Bluetooth and Wi-Fi are also measured when there is no handover.

The measurement procedure with TCP traffic load is as follows: Initially, a program named WGET on device A tries to download a file from device B, on which a FTP server is running, via Wi-Fi connection. When the program begins, a vertical handover procedure is also invoked on both devices. The total execution time has been captured. The experiment was repeated 30 times and all the transferred files were not corrupted.

The file transfer durations with the vertical handover are also compared with 
that duration when there is no handover as shown in Figure 10. Figure 10 shows that it takes $10.93 \pm 0.28$ seconds to transfer an $894 \mathrm{~KB}$ file using Bluetooth. The data rate of the Bluetooth connection is $83.88 \pm 2.07 \mathrm{KBps}$. If Wi-Fi is used, that duration is only $3.57 \pm 0.27$ seconds. The data rate of the Wi-Fi connection is $257.87 \pm 18.89 \mathrm{KBps}$. When a VHO from Bluetooth to Wi-Fi occurs, the file transfer time is $5.77 \pm 0.94$ seconds. In the inverted direction VHO, the file transfer time is $6.62 \pm 1.12$ seconds. From the collected values, the average value of $T_{W \min }$ and $T_{B \min }$ can be calculated using Equation (3) and (4), respectively:

$$
\begin{aligned}
T_{W \min } & =\left(R_{B} R_{W} T-R_{W} N\right) /\left(R_{B}-R_{W}\right) R_{W} \\
& =(83.88 * 257.87 * 6.62-257.87 * 894) /(83.88-257.87) 257.87 \\
& =1.95 s \\
T_{B \min } & =\left(R_{B} R_{W} T-R_{B} N\right) /\left(R_{W}-R_{B}\right) R_{B} \\
& =(83.88 * 257.87 * 5.77-83.88 * 894) /(257.87-83.88) 83.88 \\
& =3.41 s
\end{aligned}
$$

\subsection{Energy Efficiency Evaluation}

\subsubsection{Experiment Setup}

The energy consumption of wireless communication with and without a VHO has been measured by using a real testbed illustrated in Figure 11. On the left of the figure, the mobile device is a Linux computer (Core 2 Duo @2.26 GHz processor and 2 GB RAM, Ubuntu 14.04 64-bit). The device connects to Wi-Fi and Bluetooth networks using USB-based Wi-Fi and Bluetooth adapters, respectively. The adapters are connected to a current monitoring circuit before being attached to the mobile device. The output signal of the monitoring circuit is converted to digital signal via an analogue-to-digital converter (ADC). The digital

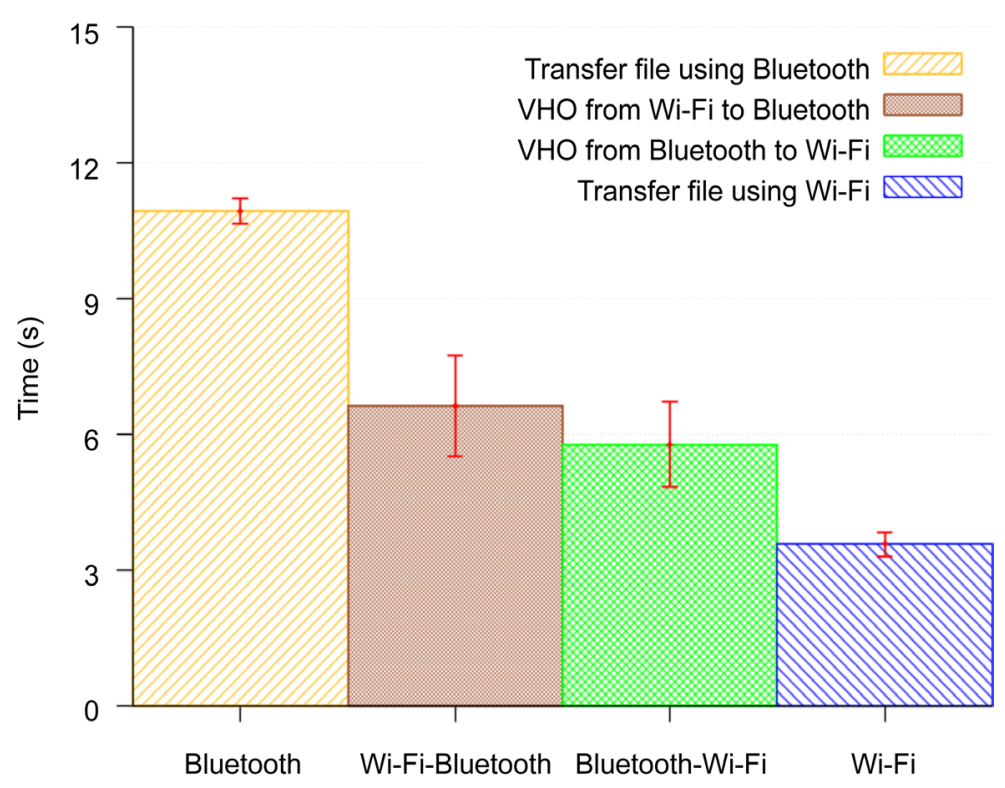

Figure 10. File transfer time. 
signal is then sent to the signal analysis device. The signal analysis device is a computer in which a small program is running to capture and save the digital signals.

\subsubsection{Energy Consumption Measurement}

In this work, each device only uses one wireless technology at almost any given time, the other technologies are turned off. A technology is called "off" when it is not attached to any network. Therefore the technology still consumes energy even it is turned off. The energy consumed in "off" state of Wi-Fi and Bluetooth has been measured and the results are given in Figure 12. As shown in the figure, the average energy consumption of Wi-Fi $\bar{P}_{W i-F i-o f f}$ and Bluetooth $\bar{P}_{\text {Bluetooth-off }}$ in the "off" state are $400.75 \mathrm{~mW}$ and $37.25 \mathrm{~mW}$, respectively.

To examine the energy consumption by each technology, device A and device $\mathrm{B}$ exchange file using FTP protocol. When two devices use Wi-Fi, Bluetooth is

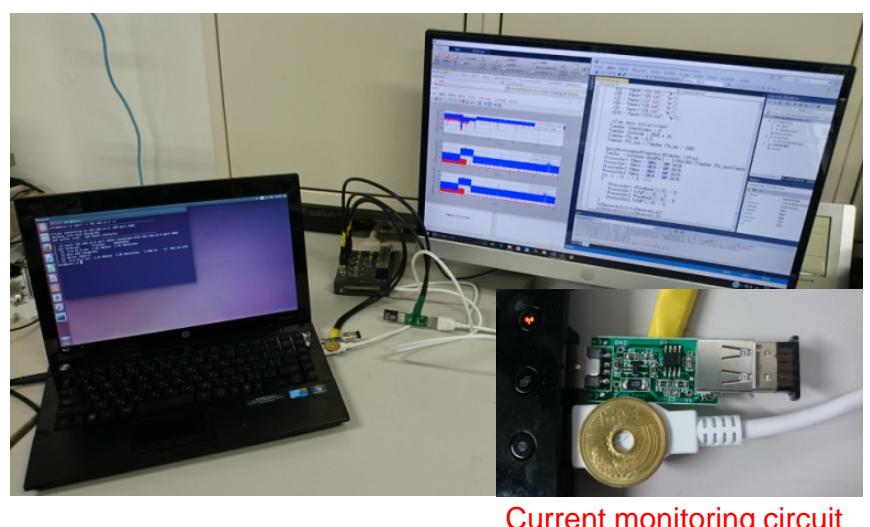

Figure 11. Energy consumption measurement setup.

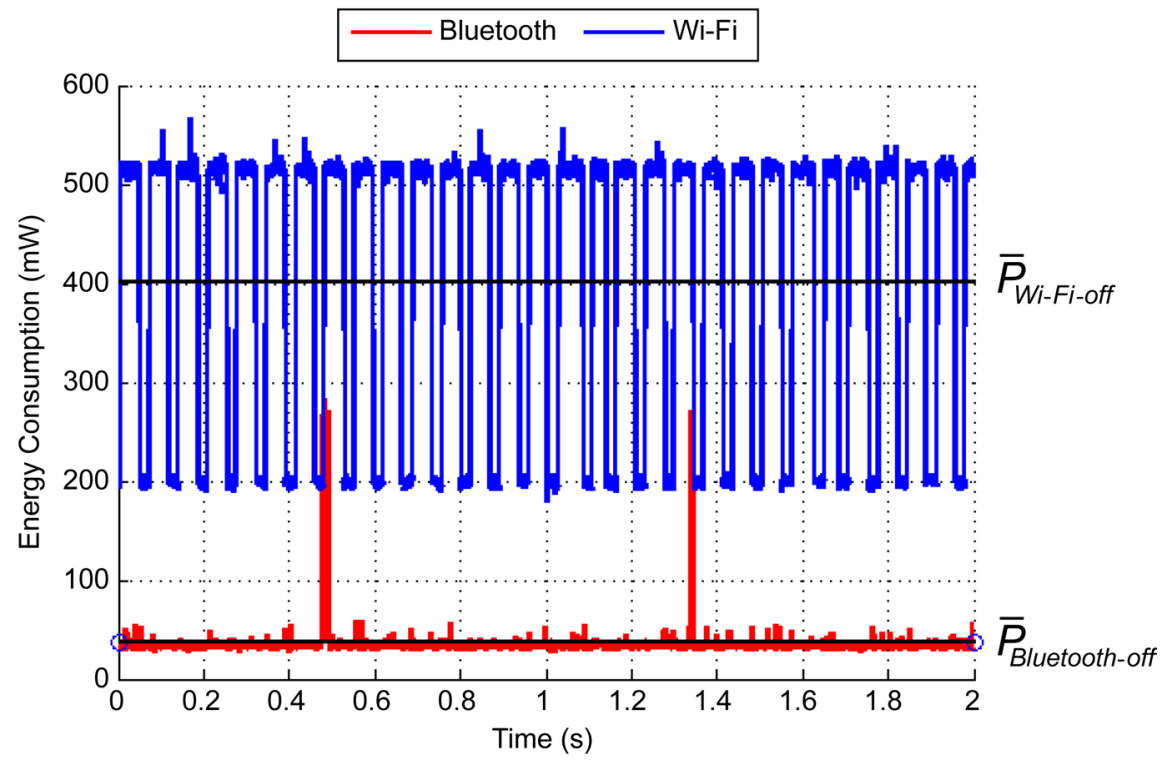

Figure 12. Energy consumption when wireless interfaces are off. 
turned off and vice versa. The measured results are shown in Figure 13 and Figure 14. Figure 13 shows the energy consumption when the device uses Bluetooth $P_{B}$. As shown in the figure, Bluetooth only consumes $82.24 \mathrm{~mW}$ on average. The total energy consumption of the wireless communication when using Bluetooth is:

$$
\bar{P}_{\text {Wireless-B }}=\bar{P}_{B}+\bar{P}_{\text {Wi-Fi-off }}=82.24+400.75=482.99 \mathrm{~mW}
$$

Similarly, Figure 14 shows the energy consumption when the device uses Wi-Fi $P_{W}$. As shown in the figure, Wi-Fi consumes $605.05 \mathrm{~mW}$ on average. The total energy consumption of the wireless communication when using $\mathrm{Wi}-\mathrm{Fi}$ is:

$$
\bar{P}_{\text {Wireless-W }}=\bar{P}_{W}+\bar{P}_{\text {Bluetooth-off }}=37.25+605.05=642.30 \mathrm{~mW}
$$

\subsubsection{Energy Saving Estimation}

Assume that a service $S$ last $T=15 \mathrm{~s}$, the average energy consumption of the wireless communication when using $\mathrm{Wi}-\mathrm{Fi}$ is:

$$
P_{W}=\bar{P}_{\text {Wireless-W }} T=642.30 * 15=9634.5 \mathrm{~mJ}
$$

The average energy consumption of the wireless communication when using Bluetooth is:

$$
P_{W}=\bar{P}_{\text {Wireless }-B} T=482.99 * 15=7244.85 \mathrm{~mJ}
$$

During the duration $T$, the maximal and minimal energy consumption by using the mobile-controlled VHO management are calculated by using Equation (6) and (7), respectively:

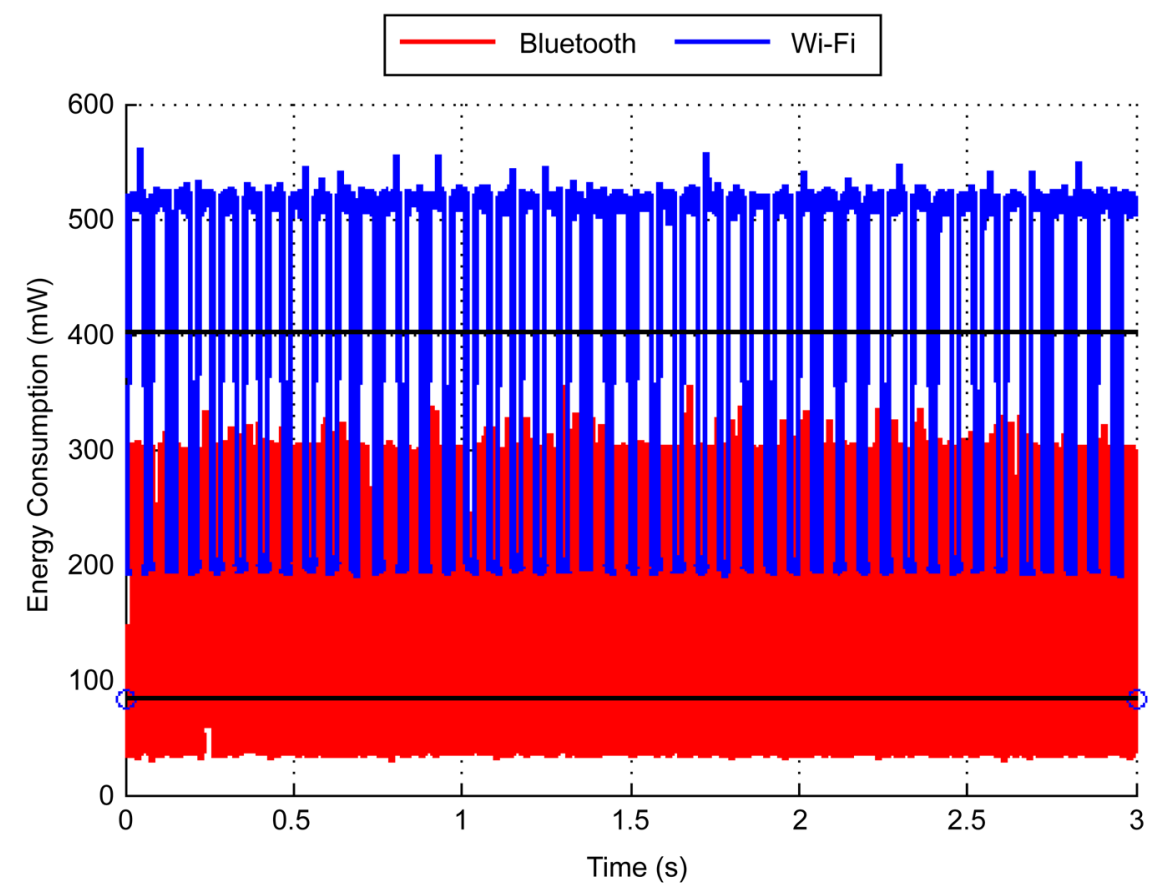

Figure 13. Energy consumption when using Bluetooth. 


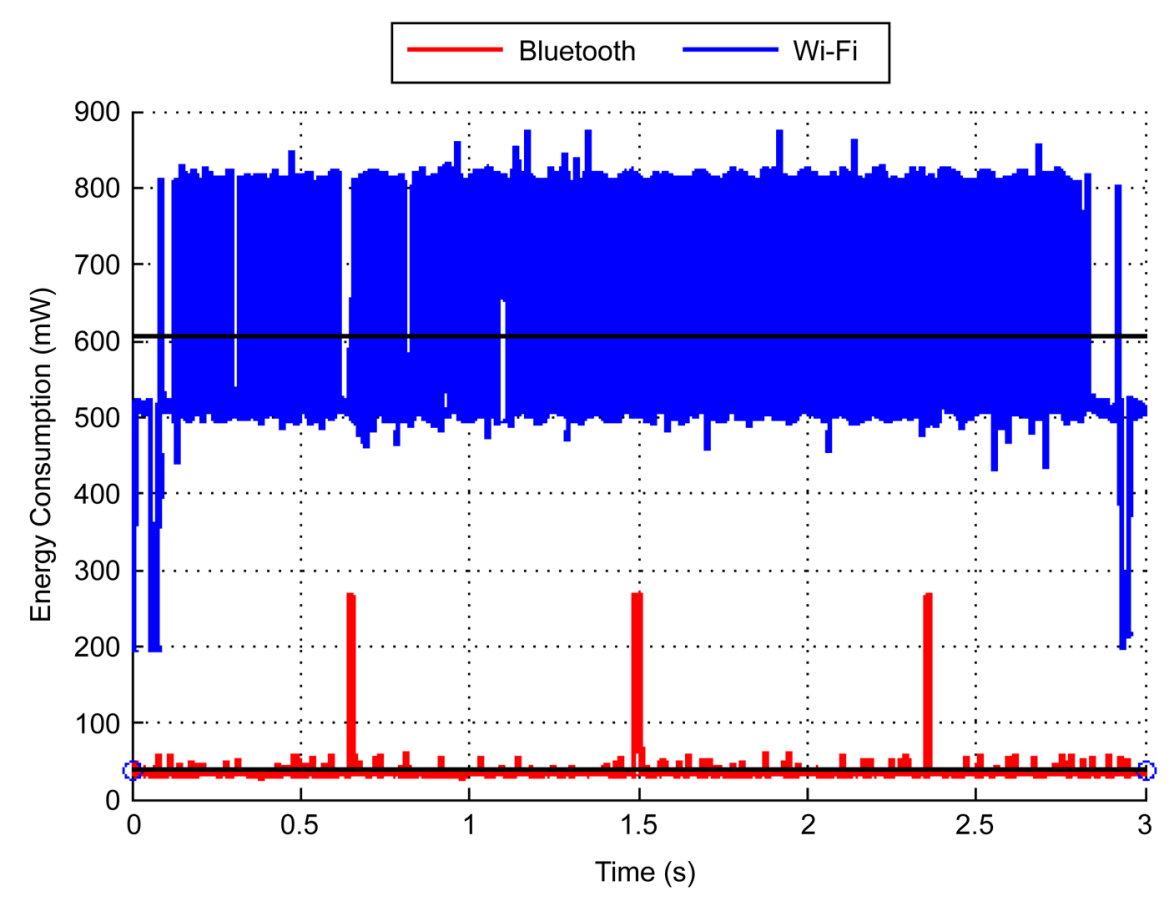

Figure 14. Energy consumption when using Wi-Fi.

$$
\begin{aligned}
P_{\min } & =\bar{P}_{\text {Wireless- } W} T_{W \min }+\bar{P}_{\text {Wireless } B}\left(T-T_{B}\right) \\
& =642.30 * 1.95+482.99(15-1.95)=7555.50 \mathrm{~mJ} \\
P_{\max } & =\bar{P}_{\text {Wireless-B }} T_{B \min }+\bar{P}_{\text {Wireless }-W}\left(T-T_{B \min }\right) \\
& =482.99 * 3.41+642.30(15-3.41)=9091.25 \mathrm{~mJ}
\end{aligned}
$$

The number of VHO can be occurred is the quotient in the division given in the Equation (8):

$$
\begin{aligned}
N_{V H O} & =\left(T /\left(T_{W \min }+T_{B \min }\right)\right) \\
& =(15 /(1.95+3.41)) \\
& =2
\end{aligned}
$$

The minimal energy consumption when there are two successful VHO occurred during the duration $T$ is calculated using Equation (9):

$$
\begin{aligned}
P_{N=2} & =\bar{P}_{\text {Wireless-W }}\left(T-N_{V H O} T_{B \min }\right)+\bar{P}_{\text {Wireless }-B} N_{V H O} T_{B \min } \\
& =642.30 *(15-2 * 3.41)+482.99 * 2 * 3.41=8548.01 \mathrm{~mJ}
\end{aligned}
$$

From the estimated results, the energy consumption by using the proposed mobile-controlled VHO management can save from $5.64 \%$ to $21.58 \%$ the energy consumed by using Wi-Fi. The results also show that, even in the worst case, multiple successful VHO can save energy at least $11.28 \%$.

\section{Conclusions}

In this paper, an energy-efficient mobile-controlled VHO management has been 
introduced. The mobile-controlled VHO is an extension of the previously proposed $\mathrm{VHO}$, aiming to support mobile devices to reduce energy consumption as well as to maintain real time services in non-interconnected heterogeneous wireless networks. The communication behavior of the system in term of message interchange has been presented and the performance of the system has been evaluated with TCP and UDP traffic load. The obtained results has confirmed that the proposed management system can maintain the continuity of real time services by offering fast handover with low packet loss rate. The energy consumption of the system has also been measured by direct measurements. Based on the measured results, the minimal and maximal energy saving has been estimated. The results show that the system can save up to $21.58 \%$ in comparison to the energy consumed by using Wi-Fi. In the worst case, the system can save only 5.64\%; however, performing multiple VHO can save more energy.

In the current work, the VHO occurs when mobile devices are communicating at fixed positions. If the devices move, their connections may be lost. Therefore, the mobility of mobile devices will be investigated as a future study.

\section{References}

[1] Gustafsson, E. and Jonsson, A. (2003) Always Best Connected. IEEE Wireless Communications, 10, 49-55. https://doi.org/10.1109/MWC.2003.1182111

[2] Feeney, L.M. and Nilsson, M.N.P. (2001) Investigating the Energy Consumption of a Wireless Network Interface in an $A d$ Hoc Networking Environment. IEEE INFOCOM, 1548-1557.

[3] Pyles, A., Qi, X., Zhou, G., Keally, M. and Liu, X. (2012) SAPSM: Smart Adaptive 802.11 PSM for Smartphones. Proceedings of the 2012 ACM Conference on Ubiquitous Computing, Pittsburgh, 5-8 September 2012, 11-20. https://doi.org/10.1145/2370216.2370219

[4] Shih, E., Bahl, P. and Sinclair, M.J. (2002) Wake on Wireless: An Event Driven Energy Saving Strategy for Battery Operated Devices. Proceedings of the 8 th Annual International Conference on Mobile Computing and Networking (MobiCom'02), New York, 23-28 September 2002, 160-171. https://doi.org/10.1145/570645.570666

[5] Lampropoulos, G., Kaloxylos, A., Passas, N. and Merakos, L. (2007) A Power Consumption Analysis of Tight-Coupled WLAN/UMTS Networks. IEEE 18 th International Symposium on Personal, Indoor and Mobile Radio Communications, 1-5. https://doi.org/10.1109/pimrc.2007.4394248

[6] An, Y.Y., et al. (2006) Reduction of Handover Latency Using Mih Services in mipv6. 20 th International Conference on Advanced Information Networking and Applications, 229-234.

[7] Perkins, C. (2002) Ip Mobility Support for ipv4. RFC Editor.

[8] Rosenberg, J., et al. (2002) Sip: Session Initiation Protocol. RFC3344. IETF. https://doi.org/10.17487/rfc3261

[9] Zimmermann, H. (1980) OSI Reference Model-The IS0 Model of Architecture for Open Systems Interconnection. IEEE Transactions on Communications COM-28, No. 4.

[10] Banerjee, N., Wu, W., Basu, K. and Das, S.K. (2004) Analysis of Sip-Based Mobility 
Management in 4g Wireless Networks. Computer Communications, 27, 697-707. https://doi.org/10.1016/j.comcom.2003.10.009

[11] Astorga, J., Aguado, M., Toledo, N. and Higuero, M. (2013) A High Performance Link Layer Mobility Management Strategy for Professional Private Broadband Networks. Journal of Network and Computer Applications, 36, 1152-1163. https://doi.org/10.1016/j.jnca.2013.01.005

[12] Nguyen-Duc, T. and Kamioka, E. (2016) A Self-Adaptive and Energy-Efficient Wireless Multi-Hop Network. 22nd Asia-Pacific Conference on Communications (APCC), Yogyakarta, 25-27 August 2016, 296-301. https://doi.org/10.1109/apcc.2016.7581510

[13] Márquez-Barja, J., Calafate, C.T., Cano, J.-C. and Manzoni, P. (2011) An Overview of Vertical Handover Techniques: Algorithms, Protocols and Tools. Computer Communication, 34, 985-997. https://doi.org/10.1016/j.comcom.2010.11.010

[14] Open vSwitch. http://openvswitch.org/

[15] Open Networking Foundation. https://www.opennetworking.org/

[16] Nguyen-Duc, T. and Kamioka, E. (2015) An Extended Sdn Controller for Handover in Heterogeneous Wireless Network. 21 st Asia-Pacific Conference on Communications (APCC), Kyoto, 14-16 October 2015, 332-337. https://doi.org/10.1109/apcc.2015.7412534

[17] Iperf Tool. http://iperf.sourceforge.net/

Submit or recommend next manuscript to SCIRP and we will provide best service for you:

Accepting pre-submission inquiries through Email, Facebook, LinkedIn, Twitter, etc. A wide selection of journals (inclusive of 9 subjects, more than 200 journals) Providing 24-hour high-quality service User-friendly online submission system Fair and swift peer-review system Efficient typesetting and proofreading procedure Display of the result of downloads and visits, as well as the number of cited articles Maximum dissemination of your research work

Submit your manuscript at: http://papersubmission.scirp.org/

Or contact jcc@scirp.org 